rect sum of simple submodules. The real difficulties are met in the attempt to enumerate the two-sided ideals which are contained in the radical.

HARVARD UNIVERSITY

\title{
ON A CONJECTURE ABOUT INFINITE CLASS FIELDS
}

\section{GEORGE WHAPLES}

If we are given any algebraic extension field, of finite degree, of a given ground field, then the $p$-adic completion of the extension field, under any one of its valuations ${ }^{1}$ (prime spots) is an algebraic extension of the completion of the ground field under the same valuation. Our original extension field (in the large) thus determines a set of algebraic extensions of $p$-adic ground fields. We shall refer to these extensions as the local components of the original field. If our extension field (in the large) is normal, then any two valuations of the extension field which induce the same valuation in the ground field determine isomorphic local components; hence in case of a normal extension field we can think of a local component as determined by a valuation of the ground field.

When our extension field is not of finite degree we must modify this definition, since the $p$-adic closure of such an extension field will in general not be algebraic over the ground field. ${ }^{2}$ For a normal extension of infinite degree we define the local component as follows: The original extension is the splitting field ${ }^{3}$ of a certain set of polynomials with coefficients in the ground field. Define the local component to be the splitting field of this same set of polynomials over the $p$-adic extension of the ground field. It is easy to show that this field is independent of the set of polynomials used (indeed, one could use the set of all polynomials of the ground field which split in the extension

Received by the editors August 12, 1946, and, in revised form, November 13, 1946.

${ }^{1}$ For theory of valuations, see E. Artin and G. Whaples, Axiomatic characterization of fields by the product formula for valuations, Bull. Amer. Math. Soc. vol. 51 (1945) pp. 469-492, and the literature cited there.

2 See Ostrowski, Über einige Fragen der allgemeine Körpertheorie, Journal für Mathematik vol. 143 (1914) pp. 225-284. I am indebted to the referee for a correction of the first version of this paper and for this reference.

${ }^{3}$ The splitting field is the smallest subfield of the algebraic closure in which all the given polynomials split into linear factors. 
field) and that it is equal to the product ${ }^{4}$ of the local components (in the original sense) of all subfields of finite degree of our original field.

We restrict ourselves here to the case where the ground field is of finite degree over the rational field and the extension field is normal. It is known that when the extension field is of finite degree, it is completely determined by its local components. ${ }^{5}$ To prove this, take any two non-isomorphic extension fields, consider one of them as ground field and their product as extension field, and apply the theorem: In any extension field there are an infinite number of primes of ground field which do not split completely.

It has been conjectured ${ }^{6}$ that the same thing is true for abelian extensions, even when they are of infinite degree. (One sees that the above proof does not work, for the field which would have to be considered as ground field would now be of infinite degree.) The purpose of this note is to disprove the conjecture. First, we observe that the conjecture is equivalent to the seemingly stronger statement: If one abelian extension includes another everywhere locally, it includes it also in the large; that is, if we are given two abelian extensions (of infinite or finite degree) of a fixed ground field, such that at every valuation of the ground field the local component of the first includes (in sense of isomorphism) the local component of the second, then the first field includes the second. To show that the second conjecture follows from the first, take two abelian extension fields which satisfy the assumption that the local component of first field everywhere includes that of second. Form the product of the two fields. The local component of the product field is everywhere the same as that of first field; hence the original conjecture would give: product field equals first fieldhence second field is subfield of first. ${ }^{7}$

However, both conjectures are false; in fact, given any finite abelian extension field, an infinite abelian extension can be constructed which includes it everywhere locally, but not in the large. Namely, let a finite abelian extension field be given. Arrange the prime spots of the ground field in some definite order. Our infinite extension field will

4 The product of a set of fields is the smallest subfield of the algebraic closure which includes them all.

5 This follows from a theorem of M. Bauer, Über einen Satz von Kronecker, Archiv der Mathematik und Physik vol. 6 (1904) pp. 218-219. For a modern, non-analytic proof of this theorem see Chevalley, La thêorie du corps de classes, Ann. of Math. vol. 41 (1940) pp. 394-418.

${ }^{6} \mathrm{C}$. Chevalley, Géneralization de la théorie de corps de classes pour les extensions infinies, Journal de Mathématiques pures et appliquées (9) vol. 15 (1936) pp. 359-371.

7 The author owes thanks to Professor Chevalley for some simplification of this part of the argument. 
be the product of a set of constituent fields, where one constituent corresponds to each prime spot of the ground field. The constituent corresponding to a given prime spot can be any finite abelian extension satisfying the conditions:

(a) Its local component includes the local component of the given field at the prime spot to which it corresponds.

(b) Each of its cyclic subfields is ramified at some place at which all the preceding fields (that is, all the fields corresponding to preceding primes) are unramified.

Grunwald's theorem ${ }^{8}$ shows that such constituent fields can be constructed. (b) can be achieved by making a product of cyclic extension fields, each of which is totally ramified at at least one place where all the fields already constructed are unramified. Condition (a) implies that the product field includes the given field everywhere locally. But we shall show that (b) implies that every cyclic subfield of our product is ramified at some place where the given field is unramified; hence given field cannot be subfield.

The proof is best carried out by means of group characters. Clearly we can replace our infinite product field by a product of a finite number of the constituents, since every cyclic subfield of the field we have constructed is contained in such a product. (The introduction of this finite subfield could be avoided by using directly the characters of the whole field; yet it seems simpler this way.)

Consider, then, any extension of our ground field which is formed by taking the product of a finite number of fields satisfying (a), (b) above. Its Galois group is abelian and finite. Each character of the Galois group determines a cyclic subfield under the correspondence: character goes into subfield of elements left fixed by the subgroup on which the character takes value 1 ; and every cyclic subfield is determined by some character. Call a character ramified (at a certain prime) if the subfield which it determines is ramified there. Clearly a character is ramified (at any spot) exactly when it is not identically 1 on the ramification group (at that spot); so it follows that, at any fixed prime spot, a product of a ramified character and an unramified character is ramified.

The characters of the constituent fields, that is, the characters which determine the fields described in (b), are evidently a basis for the group of all characters of the finite abelian group of our field.

${ }^{8} \mathrm{~W}$. Grunwald, Ein allgemeines Existenztheorem fïr algebraische Zahlkörper, Journal für Mathematik vol. 169 (1932) pp. 103-107. For a nonanalytic proof see Whaples, Nonanalytic class field theory and Grunwald's Theorem, Duke Math. J. vol. 9 (1942) pp. 455-473. 
(Indeed, condition (b) implies that this group is simply the direct product of the groups of the constituent fields.) Any character can be expressed as a product of characters determining cyclic subfields of the constituent fields; in this product, all characters belonging to the same constituent can be united into a single one, and that character which is not identically 1 and belongs to the latest of the constituents will be ramified at some spot where all the others are unramified, and where our given field is unramified.

UNIVERSITY OF WISCONSIN 